\title{
Anti-PD-1/CTLA-4 Bispecific Antibody AK104
}

National Cancer Institute

\section{Source}

National Cancer Institute. Anti-PD-1/CTLA-4 Bispecific Antibody AK104. NCI Thesaurus.

Code C153379.

\begin{abstract}
A bispecific antibody directed against the human negative immunoregulatory checkpoint receptors programmed cell death protein 1 (PD-1; PDCD1; CD279) and cytotoxic Tlymphocyte-associated antigen 4 (CTLA4; CT LA-4), with potential immune checkpoint inhibitory and antineoplastic activities. Upon administration, anti-PD1/CT LA4 bispecific antibody AK104 targets and binds to both PD-1 and CTLA4 expressed on tumorinfiltrating T lymphocytes (TILS), and inhibits the PD-1- and CT LA4-mediated downregulation of $\mathrm{T}$-cell activation and proliferation. This restores immune function and activates a sustained cytotoxic T-lymphocyte (CTL)-mediated immune response against tumor cells. Both PD-1 and CT LA4 are selectively expressed on TILs in the tumor microenvironment (TME) and negatively regulate the activation and effector functions of T-cells. They play key roles in the downregulation of the immune system and tumor evasion from host immunity. Dual checkpoint blockade of PD1 and CT LA4 with AK104 may enhance T-cell activation and proliferation more than the blockade of either immune checkpoint receptor alone.
\end{abstract}

